\title{
Management Strategies for Oral \& Maxillofacial Surgery Amid the COVID-19 Pandemic
}

\author{
Humayun Kaleem Siddiqui ${ }^{1}$ \\ Jawad Safdar ${ }^{2}$ \\ Kanza Ghauri ${ }^{3}$ \\ BDS, FCPS \\ BDS, MDS, PhD \\ BDS
}

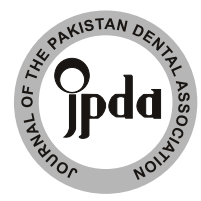

The novel-coronavirus (2019-nCOV) emerged in Wuhan City in December 2019, this resulted in a quick and catastrophic health problem all over the world but our country is slightly less affected by the pandemic. There could be a number of reasons for less numbers of Covid positive cases and lack of awareness and reduced Covid testing capacity and hence less mortality in Pakistan. In order to provide assistance to an ever increasing number of infected patients and, at the same time taking care of urgent maxillofacial conditions. This manuscript gives the reader in a nutshell the overall surgical experience of oral and maxillofacial practice at Aga Khan University Hospital, Karachi, Pakistan in the COVID-19 pandemic and would like to provide a number of recommendations that would assist the scheduling process of surgical management during the COVID-19 pandemic and reduce the risk of infection among healthcare workers and others involved with the service.

KEYWORDS: Maxillofacial surgery; Coronavirus; COVID-19; Pandemic

HOW TO CITE: Siddiqui HK, Safdar J, Ghauri K. Management strategies for oral \& maxillofacial surgery amid the COVID-19 pandemic. J Pak Dent Assoc 2021;30(4):275-278.

DOI: https://doi.org/10.25301/JPDA.304.275

Received: 02 March 2021, Accepted: 01 August 2021

\section{INTRODUCTION}

$\mathrm{T}$ he COVID 19 outbreak originated in Wuhan, Hubei province, China, and rapidly spread to other provinces and 190 other countries, was declared a global pandemic by WHO on March 9, 2020, becoming a "public health emergency of international concern". In Pakistan, first in the province of Sind followed by others imposed a strict lock down on public gatherings and limited the working hours of shops.

The patients with COVID-19 infections and are positive are the principle source of spread, the asymptomatic ones are highly infectious with a strong chance of spread in the incubation period from 1 to 14 days. The person-to- person transmission of the infection includes direct transmission from sneezing, coughing, droplets, and contact transmission, such as contact with nasal, oral, and eye mucous membranes.

The oro-faecal route yet to be explored for the spread of infection. Infection control strategies are very important to stop the virus from further spread and to help contain the

1. Consultant, Department of Oral and Maxillofacial Surgeon, Dental Section, Aga Khan University Hospital, Karachi, Pakistan.

2. Consultant, Department of Oral \& Maxillofacial Surgery, Dow Dental College, Karachi, Pakistan.

3. House Officer, Department of Oral \& Maxillofacial Surgery, Dow Dental College, Karachi, Pakistan.

Corresponding author: 'Dr. Humayhun Kaleem Siddiqui”' < humayunmxfx@ gmail.com> outbreak. The risk of spread during the diagnosis and treatment of oral diseases was also quickly assessed, adjourning non-urgent outpatient oral treatments and continuing emergent oral maxillofacial problems represented by trauma, malignant neoplasms, and infections, which require timely management.

The oral \& maxillofacial fraternity belongs to a specific group of healthcare workers as they generally come in close contact with the oral cavity, airways along with patient's secretions (for example saliva, blood, mucus,) during the diagnosis and treatment process, exposes in high risk of contracting the infection and in turn, a source of contagion. There are high viral burdens in the nasal mucosa and the viruses in aerosols that can persist for up to 3 hours in the air and 48 to 72 hours on the tops. The objective of this work is to present a working line of action while observing precautions for the patients as well as minimizing the risks pertaining to surgeons.

\section{Our Response to pandemic:}

As early as when this was intended to move toward emergency only patient management, we took guidelines from The Center of Disease Control and Prevention (USA) and The American Dental Association as well as availability of rapid scientific researches that were invaluable assets which directed our line of action. 


\section{Initial lock down}

Largely for the next four months after countrywide lockdown, maxillofacial ER patients were being admitted and if found positive, shifted to Covid ward. Although emergency surgeries were performed using full PPE with protocols, maxillofacial injuries including fractures were delayed due to increased risks of exposure and hospital policy. When they became Covid negative, only then maxillofacial fractures were managed by ORIF and IMFs. During this period, the natural flow of patients to our hospital was reduced possibly due to scarce traffic hence reduced RTAs. However, few left the hospital following recoveries and were lost to follow up.

\section{Criteria for Patient Selection during Covid Time}

Selection of patient for surgery was challenging in these times. Only selected emergencies including RTA cases, tooth abscess which need emergency incision \& drainage. To prevent corona virus infections, we followed and developed our SOPS that can help maxillofacial surgeon when they are required in emergency department.

\section{Phasic approach to operating rooms}

At first, the hospital management as well chief anesthesia decided to immediately halt all elective procedures in operating rooms allowing only emergency operations. At the end of three months and better understanding of viral transmissions, the elective cases were allowed with PPE following Covid negative tests and with guarded OR timings. Only few hours of operation theatre were allowed. Later on as the disease burden lessened, the operating rooms were opened for more elective procedures but with Covid protocols. All elective patients must have had a negative Covid PCR and mandatory use of PPE.

\section{Anesthetic and supporting staff}

Most of the anesthesia team were busy managing patients at ICUs and new Covid wards. Soon many skilled physicians including intensivists, residents, interns became the virus positive and were not readily available for elective procedures and greatly affected work. Similarly, nursing staff and the supporting staff were being called on rotations to reduce exposure and following social distancing practices. This greatly reduced the workforce necessary for maxillofacial patients.

\section{The use of personal protective equipment (PPE)}

There is scarce data which compares the utility of respirators versus surgical masks in containing the virus; however, fortunately there are a variety of published papers with discussion about the two against SARS and influenza virus. ${ }^{6}$ Wen et al tested the performance of various surgical masks and respirators and described that protection with
N95 has shown to be thirty times higher than common surgical masks. ${ }^{7}$ Moreover, the N95 superiority over surgical masks was demonstrated too when used for SARS complimenting in a series of case control researches. ${ }^{8}$ on the other hand a recent systematic review and meta-analysis did not show the superiority of N95 respirators compared to surgical masks in the course of influenza pandemics. ${ }^{9}$ This is still contradictory to the current practices against COVID-19.

To sum up, in a new challenge with the outbreak of virulent strain like COVID-19, it should be judicious to utilize respirator because it offers a seal around the nose and mouth and resistance to fluid penetration in comparison to the surgical masks and which provides protection only against droplets and similar particles including bigger respiratory droplets. The surgical masks do not provide adequate face seal and fail to filter smaller particles from the aerosols or air resulting in leakage around the mask and hence exposure to harmful droplets. Currently, the American Dental Association (ADA) recommends a quarantine of fourteen days for both health care personnel and patient if an aerosol generating procedure (AGP) was carried out using only a surgical mask and/or the patient is tested for COVID.

\section{Rate of infection in dental OPD staff and doctors}

First elective case of impacted third molars was admitted as daycare patient following hospital protocols, Covid screening and RT-PCR. Later on a strategy of Covid Pool testing was evolved and used for quick screening to reduce the economic burden on the patients and make it cost effective.

\section{DISCUSSION}

The pandemic is life threatening as well as a great mental burden on the minds of those who are disease free. In order to practice safe and prevent further spread of the disease we followed to reduce exposures to all our staff and healthcare workers. We have developed protocols along with consultation from the literature and institutional policies.

\section{Protocol for AGPs in OPDs}

Till writing of this paper, as dental, maxillofacial and ENT has extremely high chances of aerosol generation and viral loads, negative Covid-PCR are required results before any procedure. However, when rubber dam can be used patients were not tested.

\section{Protocol for AGPs in Operating Rooms}

We ensured that prior to getting into ward, the triage was carried out again and swab from nasopharynx (RT-PCR) was done both at admission followed by after 24 hours. 
Meanwhile, patients were allowed only in a separated area up to the time result arrived. A summary of flow chart can be seen in Table $2 .^{2}$

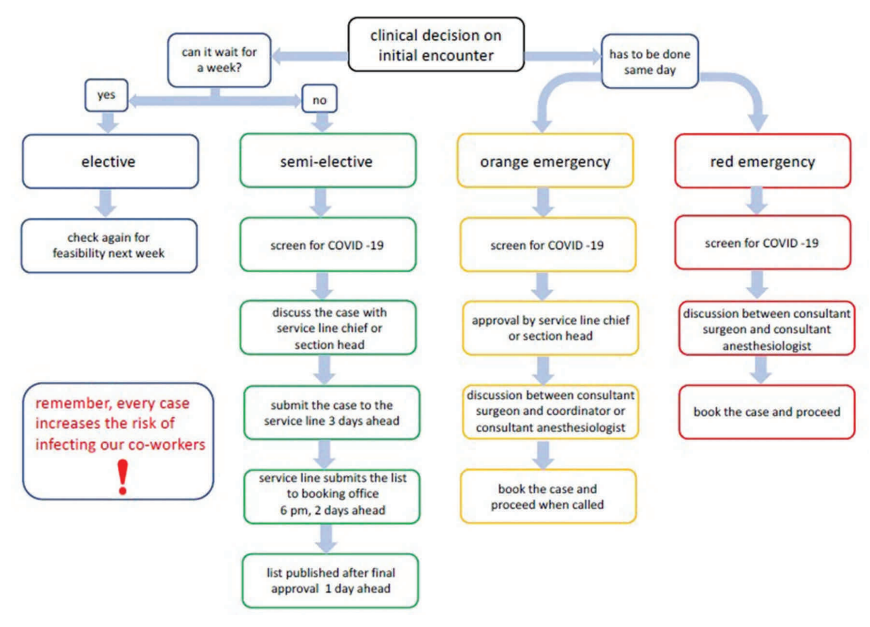

Table 1: Screening Questionnaire Used in early months of COVID crisis

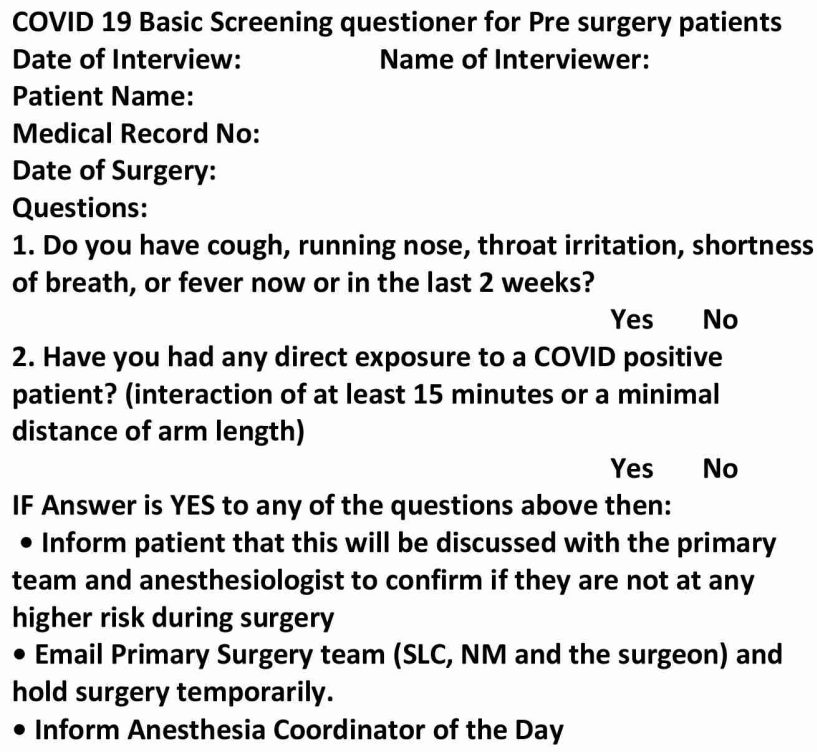

\section{Intraoperative protection}

Healthcare workers were asked to implement strict preventive strategies as directed by interim directions of WHO. All patient caring staff have used our personal protective equipment (PPE): FFP2 or N95 mask, respirators (3M), surgical gloves, fluid-resistant gown and eye protection. The total staff and materials were reduced while in the operation.

\section{Postoperative management}

The prospective patients were kept at the separated rooms. After admission they received medical management lowering use of glucocorticoids, oxygen saturation along with monitoring of vital signs. All the equipment was individualized for each patient. ${ }^{10}$ The wounds dressings were given by the paramedical staff and medical with eye protection, gloves, medical masks and surgical gowns. Patients were mobilized early so as to reduce hospital stay. The overall hospital stay was around five days apart from those who were operated for oral cancer which lasted for more than five days.

\section{Protocol after accidental exposures of peers at work or other places}

All positive coworkers and staff were required to quarantine for 14 days and tested negative before returning to work. In this matter, a unique mobile App and website is used daily by all to ensure self-screening for the symptoms. The application screens for 24 hours and gives a reminder message when expired.

\section{Home based protocols}

We used 72-hour window to operate within and patients were asked to remain inside their home until after the surgery. Covid positive patients told to quarantine and test was repeated for negative results.

\section{SOPs for Surgery}

During the period of COVID-19 emergency, commonly reported patients in the oral and maxillofacial regions were trauma and oncological diseases. The head and neck oncology team had to take care of oncology after testing negative and in some patients who were positive were operated in designated operating rooms. This included extremely necessary staff with full PPE code including N 95 or respirators. However, many elective cases of maxillofacial region were differed until end of July 2020 when the active cases declined.

All patients had negative results before surgery. Since many were admitted for daycare surgery, after successful recovery from general anesthesia were allowed to go home and remain with few attendants in the home. All the family members were explained to use surgical masks even in home when around the patient. Attendants were asked and limited to single person with protection equipment. Body temperature, heart rate, blood pressure and oxygen saturation were monitored. As all the patients had COVID-19-negative on PCR, routine preoperative tests were performed. Intraoperative barriers for healthcare staff must be strictly implemented as advised by directions of WHO.

\section{Resurgence of the active cases}

As the lockdown has eased in our country and regular 
gatherings are allowed, there are resurgence of the infected cases. Uniform protection for patients and healthcare personnel should be our priority. This may include revisiting our practices protocols, SOPs and implementation.

\section{CONCLUSION}

COVID-19 infection is a disease of concern. Strict adherence to PPE and hospital policy is required to limit and contain the virus. There is an urgent need to have a uniform policy in all the health care facilities which require resources and training of the individuals. Use of internet services including online teaching and health applications (Apps) should be used extensively to train the healthcare staff in remote areas.

\section{RECOMMENDATIONS}

The authors recommend following

1. No or minimum direct contact with the individuals.

2. Gatherings should be minimal unless necessary.

3. Where possible online mode may be used.

4. Staying home would minimize risk of exposure.

5. Improve immunity by sleeping and healthy life patterns.

6. Health care workers are responsible and should follow international as well as local governmental policies to prevent prolonged effects of deadly pandemics.

7. Regular updates on the rate of infectivity and review of institutional policies are must.

\section{CONFLICT OF INTEREST}

None to declare

\section{REFERENCES}

1. Umer F, Haji Z, Zafar K. Role of respirators in controlling the spread of novel coronavirus (COVID-19) amongst dental healthcare providers: a review. Int Endod J. 2020

https://doi.org/10.1111/iej.13313
2. Rana RE, Ather MH, Enam SA. Change in surgical practice amidst COVID 19; example from a tertiary care centre in Pakistan. Ann Med Surg (Lond). 2020;54:79-81.

https://doi.org/10.1016/j.amsu.2020.04.035

3. Government of Pakistan, See the real time Pakistan and Worldwide COVID-19 situation, [Internet]. [accessed date: 22 Apr 20] Available from: http://covid.gov.pk/.

4. American College of Surgeons, COVID-19: Guidance for Triage of Non-Emergent Surgical Procedures, [Internet]; c1996-2020 (2020) Mar 17 [cited 11 April 2020]. Available at: https://www.facs.org/covid19/clinical-guidance/triage.

5. World Health Organisation, Pakistan key indicators, [Internet]; c2016, [cited 12 April 2020]. Available at: https://apps.who.int/gho/ data/node.cco.ki-PAK

6. Umer F, Haji Z, Zafar K. Role of respirators in controlling the spread of novel coronavirus (COVID-19) amongst dental healthcare providers: a review. Int Endod J. 2020;53:1062-067.

https://doi.org/10.1111/iej.13313

7. Wen Z, Yu L, Yang W, Hu L, Li N, Wang J, Li J, Lu J, Dong X, Yin Z, Zhang K. Assessment the protection performance of different level personal respiratory protection masks against viral aerosol. Aerobiologia (Bologna). 2013;29:365-372.

https://doi.org/10.1007/s10453-012-9286-7

8. Seto WH, Tsang D, Yung RW, Ching TY, Ng TK, Ho M, Ho LM, Peiris JS; Advisors of Expert SARS group of Hospital Authority. Effectiveness of precautions against droplets and contact in prevention of nosocomial transmission of severe acute respiratory syndrome (SARS). Lancet. 2003;361(9368):1519-20. https://doi.org/10.1016/S0140-6736(03)13168-6

9. Long Y, Hu T, Liu L, Chen R, Guo Q, Yang L, Cheng Y, Huang J, $\mathrm{Du}$ L. Effectiveness of N95 respirators versus surgical masks against influenza: A systematic review and meta-analysis. J Evid Based Med. 2020;13:93-101.

https://doi.org/10.1111/jebm.12381

10. Barca I, Cordaro R, Kallaverja E, Ferragina F, Cristofaro MG. Management in oral and maxillofacial surgery during the COVID-19 pandemic: Our experience. Br J Oral Maxillofac Surg. 2020;58:687-91. https://doi.org/10.1016/j.bjoms.2020.04.025 